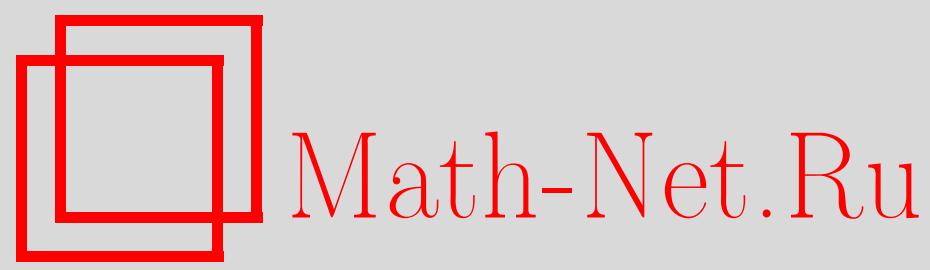

Ю. А. Фарков, Об $\varepsilon$-энтропии классов голоморфных функций, Матем. заметки, 2000, том 68, выпуск 2, 286-293

DOI: https://doi.org/10.4213/mzm945

Использование Общероссийского математического портала Math-Net.Ru подразумевает, что вы прочитали и согласны с пользовательским соглашением http://www. mathnet.ru/rus/agreement

Параметры загрузки:

IP : 54.237 .206 .68

26 апреля 2023 г., 09:32:17

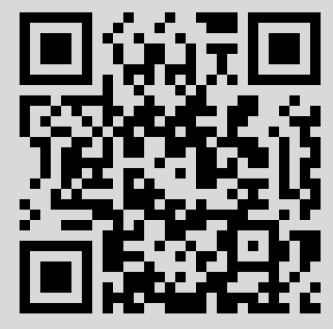


УДК 517

\section{ОБ $\varepsilon$-ЭНТРОПИИ КЛАССОВ ГОЛОМОРФНЫХ ФУНКЦИЙ}

Ю. А. Фарков

Пусть $B_{R}^{d}-$ шар радиуса $R$ в $\mathbb{C}^{d}$ и $\sigma$ - стандартная мера на единичной сфере в $\mathbb{C}^{d}$. Для $R>1,1 \leqslant p \leqslant \infty$, и натуральных $l, d$ через $H_{R}^{0}(l, p, d)$ обозначается класс голоморфных в $B_{R}^{d}$ функций $f$, у которых в однородном полиномиальном разложении первые $l$ слагаемых нулевые и радиальные производные порядка $l$ принадлежат замкнутому единичному шару пространства Харди $H^{p}\left(B_{R}^{d}\right)$. В статье получена асимптотическая формула для $\varepsilon$-энтропии класса $H_{R}^{0}(l, p, d)$ в пространствах $L^{p}(\sigma), 1 \leqslant p<\infty$, и $C\left(\bar{B}_{1}^{d}\right)$.

Библиограффия: 6 названий.

1. Формулировка результата и предварительные сведения. Пусть $A$ - предкомпактное множество в метрическом пространстве $X$ и фиксировано $\varepsilon>0$. Через $N_{\varepsilon}(A ; X)$ обозначается минимальное число точек в $\varepsilon$-сети для $A$ в $X$. Величина

$$
\mathscr{H}_{\varepsilon}(A ; X):=\log N_{\varepsilon}(A ; X)
$$

где логарифм берется по основанию 2 , называется $\varepsilon$-энтропией множества $A$ в $X$ (см., например, [1]).

Пусть $B^{d}$ - единичный шар в $\mathbb{C}^{d}, S^{d}:=\partial B^{d}$ - его граница, $B_{R}^{d}:=R B^{d}$ - шар радиуса $R, \bar{B}_{R}^{d}$-его замыкание, $\sigma$ - стандартная мера на $S^{d}, \sigma\left(S^{d}\right)=1$ (см. [2]). Пространства Харди $H^{p}\left(B_{R}^{d}\right), 1 \leqslant p \leqslant \infty$, состоят из всех голоморфных в $B_{R}^{d}$ функций $f$, имеюших конечные нормы

$$
\|f\|_{H^{p}\left(B_{R}^{d}\right)}:=\sup _{0<r<R}\left(\int_{S^{d}}|f(r \zeta)|^{p} d \sigma(\zeta)\right)^{1 / p}
$$

при $p<\infty$ и

$$
\|f\|_{H^{\infty}\left(B_{R}^{d}\right)}:=\sup \left\{|f(z)|: z \in B_{R}^{d}\right\} .
$$

Если голоморфная в $B_{R}^{d}$ функция $f$ имеет однородное полиномиальное разложение

$$
f(z)=\sum_{j=0}^{\infty} F_{j}(z), \quad z \in B_{R}^{d}
$$

Работа выполнена при финансовой поддержке Российского фонда фундаментальных исследований, гранты № 96-01-10035 и № 99-01-01181. 
то $l$-я радиальная производная этой функции определяется формулой

$$
\left(\mathscr{R}^{l} f\right)(z):=\sum_{j=l}^{\infty} \frac{j !}{(j-l) !} F_{j}(z)
$$

Для фиксированных $l, d \in \mathbb{N}, 1 \leqslant p \leqslant \infty$, через $H_{R}(l, p, d)$ обозначается класс голоморфных в $B_{R}^{d}$ функций $f$, удовлетворяющих условию $\left\|\mathscr{R}^{l} f\right\|_{H^{p}\left(B_{R}^{d}\right)} \leqslant 1$. Пусть $H_{R}^{0}(l, p, d)$ - множество функций $f$ из $H_{R}(l, p, d)$, для которых в разложении (1) первые $l$ слагаемых нулевые: $F_{0}=\cdots=F_{l-1}=0$. Положим $X_{p}:=L^{p}(\sigma)$ для $1 \leqslant p<\infty$ и $X_{\infty}:=C\left(\bar{B}^{d}\right)$.

Tеорема. Пусть $1 \leqslant p \leqslant \infty, R>1, l, d \in \mathbb{N}$. Тогда при $\varepsilon \rightarrow 0$ справедлива асимптотическая формула

$$
\begin{aligned}
\mathscr{H}_{\varepsilon}\left(H_{R}^{0}(l, p, d) ; X_{p}\right)= & \frac{2}{(d+1) !(\log R)^{d}}\left(\log \frac{1}{\varepsilon}\right)^{d+1} \\
& \quad-\frac{2 l}{d !(\log R)^{d}}\left(\log \frac{1}{\varepsilon}\right)^{d} \log \log \frac{1}{\varepsilon}+O\left(\left(\log \frac{1}{\varepsilon}\right)^{d}\right) .
\end{aligned}
$$

Некоторые аналоги формулы (2) в случае $d=1$ можно найти в [3] и [4]. Излагаемый ниже вывод этой формулы основан на известном общем методе оценок $\varepsilon$-энтропии в пространствах с базисом (см. [1, с. 277]) и результатах автора [5] о поперечниках классов $H_{R}(l, p, d)$.

Все мультииндексы $k=\left(k_{1}, \ldots, k_{d}\right)$ из $\mathbb{Z}_{+}^{d}$ занумеруем таким образом, что $k=k(j)$, $|k(j)| \leqslant|k(j+1)|, j=0,1, \ldots$; здесь, как обычно, $|k|=k_{1}+\cdots+k_{d}$. Положим $\widetilde{n}=|k(n)|, \bar{n}:=\max \{j \in \mathbb{N}:|k(j)|=|k(n)|\}, \widehat{l}:=\min \{j \in \mathbb{N}:|k(j)|=l\}$, $s_{l}:=\operatorname{card}\left\{j \in \mathbb{Z}_{+}:|k(j)|=l\right\}$.

Легко видеть, что

$$
s_{l}=\left(\begin{array}{c}
l+d-1 \\
d-1
\end{array}\right)
$$

и для $\widetilde{n}>l$

$$
\bar{n}-\widehat{l}+1=\sum_{j=l}^{\tilde{n}} s_{j}
$$

Из свойств сочетаний (см., например, [6, с. 54]) следует, что для любого натурального $N$

$$
\sum_{j=0}^{N} s_{j}=\left(\begin{array}{c}
N+d \\
d
\end{array}\right), \quad \sum_{j=1}^{N} j s_{j}=\frac{N(N+1) \cdots(N+d)}{(d-1) !(d+1)}
$$

Отметим также, что

$$
\tilde{n}=N \Longleftrightarrow\left(\begin{array}{c}
N+d-1 \\
d
\end{array}\right) \leqslant n \leqslant\left(\begin{array}{c}
N+d \\
d
\end{array}\right)-1
$$

и, следовательно, по формуле Стирлинга $\widetilde{n} \sim(d ! n)^{1 / d}, n \rightarrow \infty$. 
Для $N>l$ положим

$$
\begin{aligned}
\Phi_{N}(l, d) & :=\sum_{j=l}^{N-1} s_{j} \log [j(j-1) \cdots(j-l+1)], \\
F_{N}(l, d) & :=\frac{N^{\gamma(N, l)}}{(N-l)^{s_{l}}(N-l-1)^{s_{l+1}} \cdots 2^{s_{N-2}}},
\end{aligned}
$$

где $\gamma(N, l):=\sum_{j=l}^{N-1} s_{j}$.

Лемма 1. При $N \rightarrow \infty$ имеют место асимптотические формуль

$$
\begin{gathered}
\Phi_{N}(l, d)=\frac{l}{d !} N^{d} \log N+O\left(N^{d}\right), \\
\ln F_{N}(l, d)=\left(\frac{1}{d !}+\frac{1}{4(d-2) !}\right) N^{d}+O\left(N^{d-1} \ln N\right) .
\end{gathered}
$$

ДокАЗАТЕЛЬСТво. Для вьвода (5) достаточно воспользоваться формулами

$$
\begin{gathered}
s_{N}=\frac{N^{d-1}}{(d-1) !}+O\left(N^{d-2}\right), \quad \log [N(N-1) \cdots(N-l+1)]=l \log N+O\left(\frac{1}{N}\right), \\
\sum_{j=l}^{N-1} j^{d-1} \log j=\frac{N^{d}}{d} \log N+O\left(N^{d}\right) .
\end{gathered}
$$

Кроме того, имеем

Поскольку $N ! \geqslant(N / e)^{N}$, то

$$
\begin{aligned}
F_{N}(l, d) & =\left[\frac{N^{N-l}}{(N-l) !}\right]^{s_{l}} \prod_{j=1}^{N-l-2} \frac{N^{s_{N-j}-s_{l}}}{(N-l-j)^{s_{l+j}-s_{l}}} \\
& =\left[\frac{N^{N-l}}{(N-l) !}\right]^{s_{l}} \prod_{k=1}^{N-l-1}\left(\frac{N^{k}}{k !}\right)^{s_{N-k}-s_{N-k-1}} .
\end{aligned}
$$

где

$$
F_{N}(l, d) \leqslant e^{\gamma(N, l)}\left(\frac{N}{N-l}\right)^{s_{l}(N-l)} B_{N}(l, d)
$$

$\ln B_{N}(l, d)=\ln N \sum_{k=1}^{N-l-1}\left(s_{N-k}-s_{N-k-1}\right) k-\sum_{k=1}^{N-l-1}\left(s_{N-k}-s_{N-l-1}\right) k \ln k$.

Учитывая, что

$$
s_{N-k}-s_{N-k-1}=\left(\begin{array}{c}
N+d-k-2 \\
d-2
\end{array}\right)
$$

аналогично (5) находим

$$
\ln B_{N}(l, d)=\frac{N^{d}}{4(d-2) !}+O\left(N^{d-1} \ln N\right) .
$$

Так как ввиду (4) имеет место асимптотическое равенство

$$
\gamma(N, l)=\frac{N^{d}}{d !}+O\left(N^{d-1}\right)
$$

и $(N /(N-l))^{N-l}<e^{l}$, то из (7) следует (6). Лемма 1 доказана.

Положим $\alpha_{j}^{l}:=(j-l) ! / j !, \mathscr{P}_{N}\left(\mathbb{C}^{d}\right):=\operatorname{span}\left\{z^{k}:|k| \leqslant N, k \in \mathbb{Z}_{+}^{d}\right\}$, где $z^{k}=z_{1}^{k_{1}} \cdots z_{d}^{k_{d}}$. 
Лемма 2. Пусть $P_{N} \in \mathscr{P}_{N}\left(\mathbb{C}^{d}\right), R>1,1 \leqslant p \leqslant \infty, l, d, N \in \mathbb{N}, l<N$. Тогда имеют место оценки

$$
\left\|P_{N}(R)\right\|_{X_{p}} \leqslant R^{N}\left\|P_{N}\right\|_{X_{p}}, \quad\left\|\mathscr{R}^{l} P_{N}\right\|_{H^{p}\left(B_{R}^{d}\right)} \leqslant\left(\alpha_{N}^{l}\right)^{-1}\left\|P_{N}\right\|_{H^{p}\left(B_{R}^{d}\right)}
$$

Эти неравенства доказаны в [5].

Средние Фейера функции $f$, имеющей однородное разложение (1), определяются формулой

$$
\left(\sigma_{N} f\right)(z):=\sum_{j=0}^{N-1}\left(1-\frac{j}{N}\right) F_{j}(z) .
$$

ЛЕмма 3. Для любой функиии $f \in H^{p}\left(B_{R}^{d}\right), 1 \leqslant p \leqslant \infty$, выполнено неравенство

$$
\left\|\sigma_{N} f\right\|_{H^{p}\left(B_{R}^{d}\right)} \leqslant\|f\|_{H^{p}\left(B_{R}^{d}\right)}
$$

Действительно, для $d=1$ неравенство (8) хорошо известно, а переход к случаю $d>1$ осуществляется с помощью срез-функции.

Для $N>l$ положим

$$
\lambda_{0, k-1}^{l}=\cdots=\lambda_{l-1, N-1}^{l}=1, \quad \lambda_{j, N-1}^{l}=1-\frac{\alpha_{2 N-j}^{l}}{\alpha_{j}^{l}}\left(\frac{1}{R}\right)^{2(N-j)}, j \geqslant l .
$$

Лемма 4. Пусть $1 \leqslant p \leqslant \infty, R>1, l, d, n \in \mathbb{N}, l<\widetilde{n}$. Тогда выполнено равенство

$$
\sup _{f \in H_{R}(l, p, d)}\left\|f-\sum_{j=0}^{\tilde{n}-1} \lambda_{j, \tilde{n}-1}^{l} F_{j}\right\|_{X_{p}}=\alpha_{\widetilde{n}}^{l} R^{-\tilde{n}}
$$

əде $\left\{F_{j}\right\}$ - компоненты однородного разложсения функиии $f$.

Действительно, как показано в [5], правая часть формулы (9) совпадает со значениями колмогоровского, гельфандовского, бернштейновского и линейного $n$-поперечников класса $H_{R}(l, p, d)$ в метрике $X_{p} ;$ при этом метод

$$
f \approx \sum_{j=0}^{\tilde{n}-1} \lambda_{j, \tilde{n}-1}^{l} F_{j}
$$

является оптимальным для линейного $n$-поперечника.

2. Доказательство теоремы. Пусть $p=\infty$. Фиксируем $\varepsilon>0$ и выберем $n$ из условия

$$
\alpha_{\widetilde{n}+1}^{l} R^{-\widetilde{n}-1} \leqslant \frac{\varepsilon}{4}<\alpha_{\widetilde{n}}^{l} R^{-\widetilde{n}} .
$$

Тогда имеет место асимптотическое равенство

$$
\widetilde{n}=\frac{\log 1 / \varepsilon}{\log R}-l \frac{\log \log 1 / \varepsilon}{\log R}+O(1)
$$


В пространстве $\mathbb{C}^{\bar{n}-\widehat{l}+1}=\mathbb{R}^{2(\bar{n}-\widehat{l}+1)}$ множество $U_{n, l}$ определим условием

$$
\left(a_{k(\widehat{l})}, \ldots, a_{k(\bar{n})}\right) \in U_{n, l} \Longleftrightarrow \max _{z \in \bar{B} d}\left|\sum_{j=\widehat{l}}^{\bar{n}} a_{k(j)} z^{k(j)}\right| \leqslant 1
$$

т.e.

$$
\max _{z \in \bar{B}_{R}^{d}}\left|\sum_{j=l}^{\tilde{n}} R^{-j} \sum_{|k|=j} a_{k} z^{k}\right| \leqslant 1
$$

Множество $U_{n, l}$ выпукло в $\mathbb{R}^{2(\bar{n}-\widehat{l}+1)}$ и, следовательно, измеримо по Жордану. Введем в $\mathbb{R}^{2(\bar{n}-\widehat{l}+1)}$ меру Жордана $\mu$, нормированную условием $\mu\left(U_{n, l}\right)=1$. Если $\|\cdot\|$ - норма в $\mathbb{R}^{2(\bar{n}-\widehat{l}+1)}$, для которой $U_{n, l}$ является единичным шаром, то

$$
\left\|\left(b_{k(\widehat{l})}, \ldots, b_{k(\bar{n})}\right)\right\|=\max _{z \in \bar{B}}\left|\sum_{j=\widehat{l}}^{\bar{n}} b_{k(j)} z^{k(j)}\right| .
$$

Множество $V_{n, l}$ в $\mathbb{R}^{2(\bar{n}-\widehat{l}+1)}$ определим условием

$$
\left(b_{k(\widehat{l})}, \ldots, b_{k(\bar{n})}\right) \in V_{n, l} \Longleftrightarrow \max _{z \in \bar{B}_{R}^{d}}\left|\sum_{j=l}^{\tilde{n}}\left(\alpha_{j}^{l}\right)^{-1} \sum_{|k|=j} b_{k} z^{k}\right| \leqslant 1
$$

т.е. $\max _{z \in \bar{B}_{R}^{d}}\left|\mathscr{R}^{l} b(x)\right| \leqslant 1$, где $b(z)=\sum_{j=\hat{l}}^{\bar{n}} b_{k(j)} z^{k(j)}$.

Компоненты векторов из $V_{n, l}$ получаются умножением компонент соответствующих векторов из $U_{n, l}$ на числа

$$
\underbrace{\alpha_{l}^{l} R^{-l}, \ldots, \alpha_{l}^{l} R^{-l}}_{s_{l}}, \underbrace{\alpha_{l+1}^{l} R^{-l-1}, \ldots, \alpha_{l+1}^{l} R^{-l-1}}_{s_{l+1}}, \ldots, \underbrace{\alpha_{\widetilde{n}}^{l} R^{-\tilde{n}}, \ldots, \alpha_{\tilde{n}}^{l} R^{-\tilde{n}}}_{s_{\tilde{n}}} .
$$

Поэтому выполняется равенство

$$
\left[\mu\left(V_{n, l}\right)\right]^{1 / 2}=R^{-\sum_{j=l}^{\tilde{n}} j s_{j}} \prod_{j=l}^{\widetilde{n}}\left(\alpha_{j}^{l}\right)^{s_{j}}
$$

Пусть $\left\{g_{1}, \ldots, g_{N}\right\}$ - максимальное по числу элементов $2 \varepsilon$-различимое множество в $V_{n, l}$ (множество назьвается $\varepsilon$-различимым, если расстояние между любыми двумя его элементами больше $\varepsilon$ ). Тогда

$$
V_{n, l} \subset \bigcup_{j=1}^{N}\left(2 \varepsilon U_{n, l}+g_{j}\right), \quad \mu\left(V_{n, l}\right) \leqslant N(2 \varepsilon)^{2(\bar{n}-\widehat{l}+1)}
$$

и в силу (13)

$$
N \geqslant\left(\frac{1}{2 \varepsilon}\right)^{2(\bar{n}-\widehat{l}+1)} R^{-2 \sum_{j=l}^{\tilde{n}} j s_{j}} \prod_{j=l}^{\widetilde{n}}\left(\alpha_{j}^{l}\right)^{2 s_{j}} .
$$


Отсюда и из (3), (12) следует неравенство

$$
\mathscr{H}_{\varepsilon}\left(H_{R}(l, \infty, d), C\left(\bar{B}^{d}\right)\right) \geqslant\left(2 \sum_{j=l}^{\widetilde{n}} s_{j}\right) \log \left(\frac{1}{2 \varepsilon}\right)-2\left(\sum_{j=l}^{\widetilde{n}} j s_{j}\right) \log R+2 \sum_{j=l}^{\widetilde{n}} s_{j} \log \alpha_{j}^{l}
$$

Согласно (4) здесь

$$
\sum_{j=l}^{\widetilde{n}} s_{j}=\frac{\widetilde{n}^{d}}{d !}+O\left(\widetilde{n}^{d-1}\right), \quad \sum_{j=l}^{\widetilde{n}} j s_{j}=\frac{\widetilde{n}^{d+1}}{(d-1) !(d+1)}+O\left(\widetilde{n}^{d}\right)
$$

а последнее слагаемое в (14) оценивается с помощью (5). Послезамены $\widetilde{n}$ по формуле (11) получается требуемая оценка снизу:

$$
\begin{aligned}
\mathscr{H}_{\varepsilon}\left(H_{R}^{0}(l, \infty, d) ; C\left(\bar{B}^{d}\right)\right) \geqslant & \frac{2}{(d+1) !(\log R)^{d}}\left(\log \frac{1}{\varepsilon}\right)^{d+1} \\
& -\frac{2 l}{d !(\log R)^{d}}\left(\log \frac{1}{\varepsilon}\right)^{d} \log \log \frac{1}{\varepsilon}+O\left(\left(\log \frac{1}{\varepsilon}\right)^{d}\right) .
\end{aligned}
$$

Докажем обратное неравенство. Множества $A_{n, l}$ и $B_{n, l}$ определим условиями:

$$
\begin{aligned}
\left(b_{k(\widehat{l})}, \ldots, b_{k(\bar{n})}\right) \in A_{n, l} \Longleftrightarrow \max _{z \in \bar{B}_{R}^{d}}\left|\sum_{j=l}^{\tilde{n}}\left(\lambda_{j, \widetilde{n}}^{l} \alpha_{j}^{l}\right)^{-1} \sum_{|k|=j} b_{k} z^{k}\right| \leqslant 1, \\
\left(b_{k(\widehat{l})}, \ldots, b_{k(\bar{n})}\right) \in B_{n, l} \Longleftrightarrow \exists b_{k(\bar{n}+1)}, b_{k(\bar{n}+2)}, \ldots: \\
\max _{z \in \bar{B}_{k}^{d}}\left|\sum_{j=l}^{\tilde{n}}\left(\lambda_{j, \widetilde{n}}^{l} \alpha_{j}^{l}\right)^{-1} \sum_{|k|=j} b_{k} z^{k}+\sum_{j=\bar{n}+1}^{\infty} b_{k(j)} z^{k(j)}\right| \leqslant 1
\end{aligned}
$$

соответственно. Предположим, что полином

$$
P(z)=\sum_{j=l}^{\tilde{n}} \sum_{|k|=j} a_{k} z^{k}
$$

удовлетворяет неравенству $\|P\|_{C\left(\bar{B}^{d}\right)} \leqslant 1$. Тогда по лемме $2 R^{-\widetilde{n}} \alpha_{\widetilde{n}}^{l} P \in H_{R}(l, \infty, d)$ и согласно (9)

$$
\max _{z \in \bar{B}^{d}}\left|\left(R^{-\tilde{n}} \alpha_{\tilde{n}}^{l}\right)\left(P(z)-\sum_{j=l}^{\tilde{n}} \lambda_{j, \widetilde{n}}^{l} \sum_{|k|=j} a_{k} z^{k}\right)\right| \leqslant \alpha_{\widetilde{n}+1}^{l} R^{-\tilde{n}-1}
$$

т.е.

$$
\max _{z \in \bar{B}^{d}}\left|P(z)-\sum_{j=l}^{\tilde{n}} \lambda_{j, \widetilde{n}}^{l} \sum_{|k|=j} a_{k} z^{k}\right| \leqslant \frac{\widetilde{n}-l+1}{\widetilde{n}+1} \frac{1}{R}
$$


Отсюда следует, что

$$
\max _{z \in \bar{B} d}\left|\sum_{j=l}^{\widetilde{n}} \lambda_{j, \tilde{n}}^{l} \sum_{|k|=j} a_{k} z^{k}\right| \leqslant 2
$$

Выполнив замену переменного $z \mapsto R z$, устанавливаем, что

$$
A_{n, l} \subset 2 V_{n, l}
$$

По лемме 3 преобразование

$$
\sigma_{n}: \sum_{j=l}^{\tilde{n}} \sum_{|k|=j} a_{k} z^{k} \mapsto \sum_{j=l}^{\widetilde{n}}\left(1-\frac{j}{\widetilde{n}+1}\right) \sum_{|k|=j} a_{k} z^{k}
$$

отображает $B_{n, l}$ в $A_{n, l}$. Отсюда с учетом (16) получаем

$$
B_{n, l} \subset \sigma_{n}^{-1}\left(A_{n, l}\right) \subset 2 \sigma_{n}^{-1}\left(V_{n, l}\right)
$$

Пусть $C_{n, l}$ обозначает $\varepsilon / 2$-расширение множества $2 \sigma_{n}^{-1}\left(V_{n, l}\right)$ и пусть $\left\{x_{1}, \ldots, x_{M}\right\}-$ максимальное (по числу элементов) $\varepsilon$-различимое подмножество в $2 \sigma_{n}^{-1}\left(V_{n, l}\right)$. Тогда

$$
\bigcup_{j=1}^{M}\left(\frac{\varepsilon}{2} V_{n, l}+x_{j}\right) \subset C_{n, l}
$$

Пусть $y \in C_{n, l}$. Выберем $x \in 2 \sigma_{n}^{-1}\left(V_{n, l}\right)$ так, что $\|x-y\| \leqslant \varepsilon / 2$ (здесь $\|\cdot\|$ из (12)). В силу леммы 3 имеем $V_{n, l} \subset \sigma_{n}^{-1}\left(V_{n, l}\right)$, а по лемме 2 множество $V_{n, l}$ содержит шар радиуса $\alpha_{\widetilde{n}}^{l} R^{-\widetilde{n}}$. Значит, множество $2 \sigma_{n}^{-1}\left(V_{n, l}\right)$ содержит шар радиуса $2 \alpha_{\widetilde{n}}^{l} R^{-\widetilde{n}}$. Ввиду (10) разность $x-y$ находится в этом шаре, а с учетом вьпуклости множества $2 \sigma_{n}^{-1}\left(V_{n, l}\right)$ выполняется условие

$$
\frac{1}{2}[(y-x)+x] \in 2 \sigma_{n}^{-1}\left(V_{n, l}\right),
$$

т.е. $y \in 4 \sigma_{n}^{-1}\left(V_{n, l}\right)$. Таким образом, $C_{n, l} \subset 4 \sigma_{n}^{-1}\left(V_{n, l}\right)$ и ввиду (18)

$$
\bigcup_{j=1}^{M}\left(\frac{\varepsilon}{2} V_{n, l}+x_{j}\right) \subset 4 \sigma_{n}^{-1}\left(V_{n, l}\right)
$$

Отсюда, так как

$$
\sigma_{n}^{-1}: \sum_{j=l}^{\widetilde{n}} \sum_{|k|=j} a_{k} z^{k} \mapsto \sum_{j=l}^{\widetilde{n}}\left(\frac{\widetilde{n}+1}{\widetilde{n}-j+1}\right) \sum_{|k|=j} a_{k} z^{k}
$$

находим

$$
\sqrt{M} \leqslant\left(\frac{8}{\varepsilon}\right)^{\widetilde{n}-\widehat{l}+1} \frac{(\widetilde{n}+1)^{\tilde{n}-\widehat{l}+1}}{(\widetilde{n}-l+1)^{s_{l}(\widetilde{n}-l)^{s_{l+1}} \cdots 2^{s_{\tilde{n}}-1}}}\left[\mu\left(V_{n, l}\right)\right]^{1 / 2} .
$$


Далее, согласно (3), (6) и (13) имеем асимптотическую оценку

$$
\log M \leqslant\left(2 \sum_{j=l}^{\tilde{n}} s_{j}\right) \log \frac{1}{\varepsilon}-2\left(\sum_{j=l}^{\tilde{n}} j s_{j}\right) \log R+2 \sum_{j=l}^{\tilde{n}} s_{j} \log \alpha_{j}^{l}+O\left(\widetilde{n}^{d}\right) .
$$
номы

Пусть $x_{m}=\left(b_{k(\widehat{l})}^{(m)}, \ldots, b_{k(\bar{n})}^{(m)}\right), m=1, \ldots, M,-$ векторы из (18). Покажем, что поли-

$$
P_{m}(z)=\sum_{j=l}^{\tilde{n}} \sum_{|k|=j} b_{k}^{(m)} z^{k}, \quad k=1, \ldots, M,
$$

образуют $5 \varepsilon / 4$-сеть для $H_{R}^{0}(l, \infty, d)$ в пространстве $C\left(\bar{B}^{d}\right)$. Для любой $f \in H_{R}^{0}(l, \infty, d)$ в силу (9) и (10) имеем оценку

$$
\max _{z \in \bar{B}}\left|f(z)-\sum_{j=l}^{\tilde{n}} \lambda_{j, \widetilde{n}}^{l} F_{j}(z)\right| \leqslant \alpha_{\widetilde{n}+1}^{l} R^{-\tilde{n}-1} \leqslant \frac{\varepsilon}{4},
$$

где $F_{j}(z)=\sum_{|k|=j} \hat{f}_{k} z^{k}$. Так как

$$
\left\|\mathscr{R}^{l} f\right\|_{H^{\infty}\left(B_{k}^{d}\right)}=\sup _{z \in B_{k}^{d}}\left|\sum_{j=l}^{\infty}\left(\alpha_{j}^{l}\right)^{-1} F_{j}(z)\right| \leqslant 1,
$$

то вектор $f_{n, l}:=\left(\lambda_{l, \widetilde{n}}^{l} \hat{f}_{k(\widehat{l})}, \ldots, \lambda_{\widetilde{n}, \widetilde{n}}^{l} \hat{f}_{k(\bar{n})}\right)$ принадлежит $B_{n, l}$. Ввиду $(12)$ и $(17)$ среди $x_{1}, \ldots, x_{M}$ найдется вектор $x_{m}$ такой, что

$$
\left\|f_{l, n}-x_{m}\right\|=\left\|\sum_{j=l}^{\tilde{n}} \lambda_{j, \widetilde{n}}^{l} F_{j}-P_{m}\right\|_{C\left(\bar{B}^{d}\right)}<\varepsilon .
$$

Но тогда $\left\|f-P_{m}\right\|_{C\left(\bar{B}^{d}\right)}<5 \varepsilon / 4$. Поэтому

$$
\mathscr{H}_{5 \varepsilon / 4}\left(H_{R}^{0}(l, \infty, d) ; C\left(\bar{B}^{d}\right)\right) \leqslant \log M
$$

Отсюда и из (19) с помощью (4), (5) и (11) получается оценка, обратная (15). В случае $1 \leqslant p<\infty$ формула (2) устанавливается аналогично. Теорема доказана.

\section{СПИСОК ЦИТИРОВАННОЙ ЛИТЕРАТУРЫ}

[1] Тихомиров В. М. Некоторые вопросы теории приближений. М.: Изд-во МГУ, 1976.

[2] Рудин У. Теория функций в единичном шаре из $\mathbb{C}^{n}$. М.: Мир, 1984.

[3] Двейрин М.З. Поперечники и $\varepsilon$-энтропия классов функций, аналитических в единичном круге // Теория функций, функцион. анализ и их прилож. Вып. 23. Харьков: Изд-во Харьк. ун-та, 1975. С. 32-46.

[4] Фарков Ю. А. О поперечниках некоторых классов аналитических функций // УМН. 1984. T. 39. № 1. C. 161-162.

[5] Farkov Yu. A. The $N$-widths of Hardy-Sobolev spaces of several complex variables // J. Approx. Theory. 1993. V. 75. №2. P. 183-197.

[6] Виленкин Н.Я. Комбинаторика. М.: Наука, 1969. 